\title{
SOMATIC MARKERS IN DECISION-MAKING WITH REGARD TO THE COGNITIVE DEVELOPMENT OF 6- TO 14-YEAR-OLD CHILDREN
}

\author{
Mária TÚNYIOVÁ, Ivan SARMÁNY-SCHULLER \\ Institute of Experimental Psychology \\ Centre for Social and Psychological Sciences, Slovak Academy of Sciences \\ Dúbravská cesta 9, 84104 Bratislava, Slovak Republic \\ E-mail: maria.tunyiova@savba.sk
}

\begin{abstract}
The aim of the research was to study somatic markers during decision-making in children aged 6-14 years. On the basis of the success in Piaget's experiments, 27 children were divided into three groups - preoperational stage, concrete operational stage and formal operational stage. Children played a modified Iowa Gambling Task while skin conductance and heart rate were measured. The somatic markers appeared during decision-making in children from the age of approximately 10 years, the period of concrete operational stage.
\end{abstract}

Key words: somatic markers, Iowa Gambling Task, Ineco Children Gambling Task (ICGT), cognitive development - Piaget concept, children 6-14 yrs. old

\section{Introduction}

Emotional states are accompanied by physiological changes in the body mediated by peripheral, autonomic, endocrine, and skeletomotor responses (Iversen, Kupfermann, \& Kandel, 2000). These physiological changes are based on past experiences, when the body "learned" that a certain event leads to a pleasant or unpleasant feeling. Damasio (1996) called them somatic markers. Although somatic markers are often unconscious (e.g., pupil dilation), the

Acknowledgment

The authors would like to thank Prof. Dr. Gerhard Stemmler and Prof. Dr. Jan Wacker for helpful comments to the first draft of the manuscript. This research was supported by the VEGA grant agency with number 2/0116/15. brain evaluates them as relevant information, which we perceive as an intuitive feeling. Bechara et al. (1997) found that emotion experienced in decision-making (e.g., during risky choices) is accompanied by somatic physiological changes.

The aim of our research was to determine whether somatic markers, which reflect intuitive feelings and which dissuade adults from risky choice, occur also during decision-making in children. Skin conductance is considered a primary indicator of somatic markers, in particular of sympathetic physiological arousal (Bechara et al., 1997). Healthy people generate skin conductance responses (SCR) whenever they consider risky behavior and the SCR occurs even before they explicitly know about the riskiness of a choice. Furthermore, SCRs are larger during anticipation of losses than during anticipation of gains (Bechara et al., 1996).

DOI: $10.21909 /$ sp.2016.01.705 
Heart rate (HR) is another indicator of anticipatory physiological arousal. HR slows down when a person prepares for a response, and when the consequence is likely to be disadvantageous and risky, HR slows down even more (Crone et al., 2004).

The Iowa Gambling Task (IGT, Bechara, 2007) is often employed to investigate decision-making and somatic markers. A participant's task in the IGT is to select cards from four decks to collect as much money as possible. Some cards are risky, because they lead to losses in the long run (despite large shortterm gains), and others are less risky, because they lead to gains. Successful solving of IGT requires sensitivity to one's own emotions or intuition (Pilárik \& Sarmány-Schuller, 2009).

Men perform better in the IGT and the differences between men and women in IGT task progression and performance are small but consistent, often only shown in large samples of participants (van den Bos, Homberg, \& de Visser, 2012). This difference has been demonstrated in children and adolescents as well (Overman et al., 2004; Crone et al., 2005).

After Bechara et al. (1996), Damasio (1996) and Crone et al. (2005) reported the failure of children in the IGT, they concluded that children are not "sensitive" to consequences due to the "absence of emotional somatic markers" related to choosing the advantageous option and avoiding the risky one. Crone et al. (2005) believe that the inability of children (aged 7-12 years) to choose the objectively advantageous option is due to the later maturation of the prefrontal cortex. They compared the decision-making of children in IGT to Bechara's sample of patients with lesions in the ventromedial prefrontal cortex, who also tend to fail in the IGT
(Bechara et al., 1994). However, Crone et al. (2005) did not directly measure somatic markers.

On the other hand, Crone, Vendel, and Van der Molen (2003) claim that healthy people aged 12-25 years are already able to successfully deal with IGT and their success is dependent on the age increase. Research of Hooper and her colleagues (Hooper et al., 2008), focused on adolescents, shows that children aged 9-17 years are able to successfully deal with IGT and also that the success was positively correlated with age of participants.

Four decks in the IGT are characterized by three dimensions - frequency of loss, magnitude of loss, and certain gain. Each of the decks represents a different ratio of parameters of each dimension. For the success in the IGT it is necessary to understand the relations between all dimensions, and also parameters of one deck related to another one, to find out which deck is more or less risky.

Child's thinking in the preoperational stage (PS) (age 2-7 years) is inter alia characterized by focusing only on one aspect of a situation and ignoring other, possibly much more significant ones. Piaget (1976) called this phenomenon "centration". It is therefore likely that children in this stage will not be able to simultaneously take into account all the dimensions of each card deck of the IGT. We assume that children in the PS will focus only on one dimension in the IGT and this will be the ratio of rewards and punishments. Therefore, they should prefer a risky option with a low frequency of large losses.

Successful solution of the IGT requires understanding the relationships that are relatively abstract. Children in the concrete operational stage (COS) (7-11 years) can oper- 
ate only with symbols of (concrete) physical objects and activities in their mind (Piaget, 1976). We therefore assume that children in the COS will focus mainly on the frequency of wins and losses, which does not require an abstract understanding of the relationship between all three dimensions. Therefore, we predict that they will prefer the deck containing more frequent rewards and less frequent losses irrespective of the magnitude of the losses.

In the formal operational stage (FOS) (age 11-15 years) the reasoning of children changes from concrete to abstract and they are able to combine and match various elements of a problem (Piaget, 1976). Therefore, we assume that those children will be able to grasp all parameters of the decks and the relationships between them and this is the reason why they will be more successful in the IGT, compared to children of previous stages.

It is known that somatic changes appear depending on the expected consequences of the particular decision. We expected greater somatic responses after the choices (before feedback) that were considered as more risky by children of different cognitive stages. Because an objectively advantageous choice, which is much too complex, is likely to be seen as risky by young children, we expected greater somatic responses after both risky and complex advantageous choices by children in the PS and COS.

\section{Methods and Sample}

\section{Sample}

We studied decision-making and psychophysiological changes in 27 children aged
6-14 years. The lower age limit was set to include first grade students, who already know the basics of mathematics. All children were from the majority population and from middle class families from a town with approximately 13000 citizens.

Children were divided into three groups according to their stage of cognitive development. The criterion for inclusion into one of the three groups was the successful or unsuccessful solving of Piaget's tasks. We verified the ability of conservation of volume (children's task was to determine whether the volume of water stays the same regardless of the shape of the vessels) and conducted the experiment with the pendulum (three different weights and three different length of strings were available for use to construct the fastest and slowest pendulum - the task can test abstract thinking and the ability to perceive a number of factors, the relationship between them and the ability to select one relevant factor across the problematic situation). 7 children (6 girls, 1 boy, $M$ age $=7.5$ years, $S D=1.08$ ) were in the PS, 10 children ( 7 girls, 3 boys, $M$ age = 10.45 years, $S D=2.49$ ) were in the COS, and 10 children $(5$ girls, 5 boys, $M$ age $=12.25$ years, $S D=2.04$ ) were in the FOS.

\section{Modified IGT}

Children played an experimental gambling task, which was modified relative to the original IGT (Bechara, 2007). The subject's task was to select cards trying to win as much fictitious Iowa dollars as possible. The modification was prepared according to the model of the modified version of IGT from the Institute of Cognitive Neurology in Buenos Aires (INECO). The modified version of IGT is called Ineco Children Gam- 
bling Task (ICGT) (Túnyiová et al., 2012). The ICGT was in the form of a computer game programed in Presentation (Neurobehavioral Systems) and modified from the IGT as follows:

1) The original IGT was divided into two parts as follows:

- In the first part of ICGT (ICGT1) children had to choose cards from only two decks, which differed only in two parameters - the magnitude of gains (which differs between decks and is constant) and the frequency of losses. The magnitude of eventual losses in both decks was approximately the same.

- Bechara et al. (1997) observed a change in the SCR in healthy people starting in the range of 11 th to 20 th card selection. Therefore, we considered allowing only 40 card selections. Another reason for reducing card selections from 100 to 40 is our assumption that in the ICGT1 it is easy to find the advantageous deck.

- In the second part of ICGT (ICGT2) it was necessary to take into account the proportion of three variables - the magnitude of gains, the frequency of losses, and the magnitude of losses (in this case the magnitude of losses varies between two decks).
- The ICGT2 was more challenging. Therefore, we decided to allow 60 card selections.

2) The currency was changed to Euros and the amount of money was decreased so that wins at cards were 2 or $4 €$, and possible losses were from 1 to $60 €$, the initial amount of money was $150 €$.

If a child chose cards from only the risky deck, he or she lost $10 €$ in total after 10 card selections. On the other side, 10 card selections from the advantageous deck lead to a win of $10 €$ in total (Table 1).

The ICGT1 contains only two parameters, therefore it is quite likely for young children to be successful in the game. In the ICGT2 the advantageous deck entails a high percentage of losses of small magnitude. The risky deck is attractive, because the number of losing cards is small; they are, however, associated with large losses (often more than half of the current total of gains). Deviating from the original IGT allowed us to gradually increase the difficulty of the task. In this way we could determine the degree of complexity, which children of different ages were able to assess in order to make profitable decisions.

Table 1 Characteristics of the risky and advantageous decks in the ICGT1 and ICGT2

\begin{tabular}{lcccc}
\hline & \multicolumn{2}{c}{ ICGT1 } & \multicolumn{2}{c}{ ICGT2 } \\
\hline & Risky deck & $\begin{array}{c}\text { Advantageous } \\
\text { deck }\end{array}$ & Risky deck & $\begin{array}{c}\text { Advantageous } \\
\text { deck }\end{array}$ \\
Gain per card & $4 €$ & $2 €$ & $4 €$ & $2 €$ \\
Average loss per 10 cards & $50 €$ & $10 €$ & $50 €$ & $10 €$ \\
Score per 10 cards & $-10 €$ & $10 €$ & $-10 €$ & $10 €$ \\
$\begin{array}{l}\text { Number of losing cards per } \\
10 \text { cards }\end{array}$ & 5 & 1 & 1 & 5 \\
\hline
\end{tabular}


Children played ICGT on a computer and were asked to select cards and to try to win as much money as possible; and if they failed to win, they should try to avoid losses as much as possible. Children knew that one deck was advantageous and the other was a risky one. They had to find out which deck was more advantageous and then continue to take cards from that deck.

\section{SCR and HR Measurement}

SCR and HR wererecorded using the Biopac Student Lab System. We used the MP36 data acquisition unit. In the case of HR measurement, we used disposable self-adhesive Ag/ $\mathrm{AgCl}$ snap electrodes and general-purpose electrode lead SS2LB. Two electrodes were positioned at the medial part of the ankle and the ventral part of the wrist of the left hand. $\mathrm{SCR}$ was measured with reusable $\mathrm{Ag} / \mathrm{AgCl}$ electrodes filled with a 0.05 molar sodium chloride Unibase emulsion. Two electrodes were attached at the distal phalanx of index and middle finger of the left hand.

The HR and electrodermal activity (EDA) signals were sampled at $1000 \mathrm{~Hz}$. The EDA signals were low-pass filtered at $66.5 \mathrm{~Hz}$ and the HR signals were band-pass filtered at 0.5 to $35 \mathrm{~Hz}$ to remove unwanted frequency components. Furthermore, after visual inspection we removed all segments with motion artifacts from the analysis.

A typical criterion is that the onset of an SCR has to be between 1 and $3 \mathrm{~s}$ after stimulus onset (Figner \& Murphy, 2011). In earlier studies of somatic markers the SCR is researched before a card selection or also after the feedback (Bechara et al., 1996). Bechara et al. (2005) observed SCR already 5 seconds before a card selection was manually made. $\mathrm{SCR}$ is reactional increase of arousal, which starts to occur approximately 1 second after the stimulus and reaches its peak approximately in the 3rd second (Figner \& Murphy, 2011). If the peak is observed 5 seconds before the card selection, it means that the event to which the peak binds happened about 8 seconds before the card selection. But it is questionable whether the final decision of the respondent happened already in the 8th second before pressing the keyboard and the respondent did not consider any other choice. In earlier studies the SCR index emotional arousal during the period in which the participant deliberates the choices. Hinson et al. (2006) say that it is not possible to know how many different choices were actually being deliberated in this period, only that a particular choice was ultimately made. There are some newer studies which have examined the interval after the card selection and before feedback (Tchanturia et al., 2007). We decided to examine somatic markers in that interval too, because in our opinion it better reflects increasing arousal related to the anticipation of the outcome of the concrete decision made.

We were interested in SCR and HR related to the card selection from the risky deck and from the advantageous deck separately. Because SCR related to the card selection should appear 1-2 seconds after the selection, we set a 4-second interstimulus interval after a card selection and before a feedback. The feedback meant turning of the selected card, when the child could see the amount of money - won or lost. After the feedback a 6-second interstimulus interval ensued during which the SCR was expected as a physiological response to feedback. We did not measure the SCR after feedback, this served as the minimum time interval for SCR to return approximately to the baseline. 
- The SCR was measured in units of microsiemens and reflects the peak-to-peak amplitude (highest minus lowest value) of the EDA in the observed period. Then we averaged artifact-free EDA segments after the card selection from the risky and advantageous deck respectively to obtain mean SCR of risky and advantageous deck.

- The HR was measured in units of "beats per minute" and reflects the average HR during the observed period after the card selection from the risky and advantageous deck, respectively.

We measured both HR and SCR in children in the PS, however due to the numerous motion artifacts in skin conductance recordings we decided not to analyze the SCR data in children in the PS.

The performance in the ICGT1 and ICGT2 was evaluated according to the formula: score $=$ (sum of cards drawn from the "advantageous deck") minus (sum of cards drawn from the "risky deck"). We divided total card selections into blocks and one block represented twenty card selections.

\section{Results}

In all the following statistical analyses we set the level of statistical significance to $p=$ 0.05 .

At first we conducted intersexual comparison of the score of ICGT1 (Mann-Whitney $\mathrm{U}$ test); changes in SCR between 2 blocks ( $2 \times 2$ repeated measures ANOVA (SCR values for each of 2 decks $\times 2$ blocks), sex as the between-subject factor), HR (2x2 repeated measures ANOVA (HR values for each of 2 decks $x 2$ blocks), sex as the between-subject factor) in each of Piaget's stage separately. We conducted the same procedure with ICGT2 data too.
We did not find differences between boys and girls in the score in either of the ICGT games and not in the changes of SCR and HR either. Therefore, the following statistical analysis is conducted regardless of sex differences of participants.

\section{Description}

Since the logs of children in the PS contained more than $50 \%$ of motion artefacts, we decided not to analyze data from SCR in this group. In the group of children in the COS, we observed approximately $10 \%$ artefacts and in the group of children in the FOS approximately $3 \%$, but the artefacts were randomly located throughout the record. Therefore, we decided to analyze SCR logs in those groups. In Table 2 are description statistics of scores, HR and SCR of each group, of each block. The HR and SCR were measured for advantageous and risky deck separately.

According to mean scores in the description (Table 2), in the ICGT1 children in the COS and the FOS soon found out which deck was advantageous and they achieved a positive score already in the first block $(M=5.6$ in COS; $M=4.5$ in FOS). Children in the PS achieved a positive score, however, the score was low $(M=0.57$ in the first block and $M=$ 2.29 in the second).

In the ICGT2 children in the PS preferred the risky deck and therefore achieved a negative score ( $M=-9.71$ in the third block). Their preference for risky choice was relatively stable. Children in the COS preferred risky choice at first ( $M=-3.4$ in the first block), but in the third block achieved a higher (although still negative) score $(M=-1)$. Children in the FOS seemed to be confused at first $(M=0.8)$, but in the third block they started to prefer the advantageous deck $(M=6.2)$. 
Table 2 Descriptive statistics of scores, HR and SCR of each deck, each block and each group

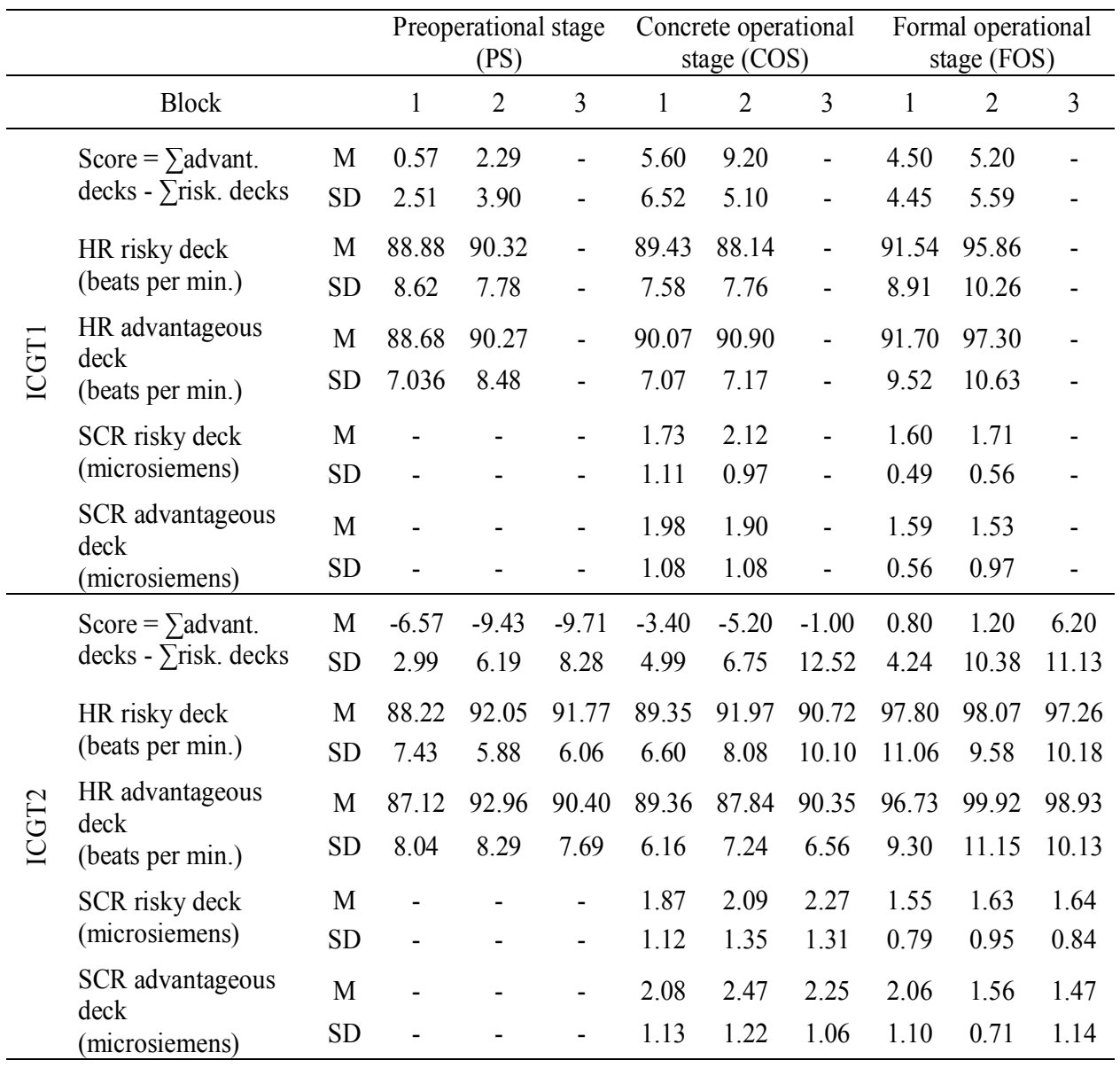

\section{Statistical Analysis}

\section{Performance in ICGT1 and ICGT2}

According to descriptive statistics, groups of children played the games differently. For analysis of differences in the achieved score in each block among the three groups we opted for the one-way ANOVA with the Piaget's stage as the Group factor (Table 3).

The groups differed among themselves in the achieved scores in almost every block. The most successful in the second block in the ICGT1 $(F(2,24)=4.060, p<0.05)$ were children in $\operatorname{COS}(M=9.2)$. In the ICGT2 in all 
Table 3 Comparison of difference in achieved score in each block between the three groups

\begin{tabular}{lllr}
\hline & & F & Sig. \\
\hline 0 & Score in block 1 & $(2,24) 2.206$ & 0.132 \\
& Score in block 2 & $(2,24) 4.060$ & 0.030 \\
\hline & Score in block 1 & $(2,24) 6.339$ & 0.006 \\
& Score in block 2 & $(2,24) 3.662$ & 0.041 \\
\hline
\end{tabular}

Table 4 Comparison of achieved score between blocks within each group separately. Comparison of increasing/decreasing score within blocks between groups

\begin{tabular}{llcc}
\hline & & $F$ & Sig. \\
\hline & PS - score between blocks 1-2 & $(1,6) 0.720$ & 0.429 \\
& COS - score between blocks 1-2 & $(1,9) 2.761$ & 0.131 \\
& FOS - score between blocks 1-2 & $(1,9) 0.393$ & 0.546 \\
& score between blocks x P. stage & $(2,24) 0.732$ & 0.492 \\
\hline & PS - score between blocks 1-2-3 & $(2,12) 0.950$ & 0.414 \\
$\underbrace{}_{0}$ & FOS - score between blocks 1-2-3 & $(2,18) 1.240$ & 0.313 \\
& FOS - score between blocks 1-2-3 & $(2,18) 1.229$ & 0.316 \\
\hline
\end{tabular}

Note. PS - Preoperational stage, COS - Concrete operational stage, FOS - Formal operational stage

blocks the most successful were children in FOS. In the first block $(F(2,24)=6.339, p<$ $0.05)$ they achieved a score of $M=0.80$; in the second block $(F(2,24)=3.661, p<0.05)$ $M=1.20$, in the third block $(F(2,24)=4.272$, $p<0.05), M=6.2$.

The mean values in Table 2 show that the score changed between the blocks. For proofing the significance of this change within each group separately and between groups we conducted repeated measures ANOVA with within-subject factor score in block 1 , block 2 , and eventually block 3 in the ICGT2. Piaget's stage represented the between-subjects factor (Table 4).

According to results from Table 4 it seems that in the groups the score did not increase or decrease significantly during the game. There was no significant difference in changes in the score between groups either. 
If we focus on the comparison of scores among groups at one time, groups differed how they performed in individual blocks (Table 3). However, the performance of each group did not change significantly between blocks in ICGT1 or ICGT2 (Table4). It is probably because children figured out relatively quickly the strategy of the game and did not change it significantly while playing.

\section{Somatic Markers}

HR slows down more when a person expects disadvantageous consequences and the SCR increases more when making a risky decision rather than an advantageous one. Focusing on changes between blocks in anticipatory HR and SCR associated with a risky or an advantageous deck, we conducted the repeated measures ANOVA (within-subject factor is mean $\mathrm{HR}$ or SCR values of each deck and of each block separately). We were also interested whether there is a significant difference in those changes of anticipatory $\mathrm{HR}$ or SCR among groups (within-subject factor is the mean HR or SCR values of each block, between-subject factor is the Piaget's stage) (Table 5).

Table 5 Comparison of HR and SCR between blocks within each deck separately and comparison with Piaget's stage

\begin{tabular}{lrr}
\hline & $F$ & Sig. \\
\hline HR of risky deck between blocks 1-2 & $(1,23) 10.553$ & 0.004 \\
HR of risky deck between blocks 1-2 x P. stage & $(2,23) 13.986$ & 0.000 \\
HR of advantageous deck between blocks 1-2 & $(1,24) 10.250$ & 0.004 \\
HR of advantageous deck between blocks 1-2 x P. stage & $(2,24) 4.151$ & 0.028 \\
SCR of risky deck between blocks 1-2 & $(1,18) 1.575$ & 0.226 \\
SCR of risky deck between blocks 1-2 x P. stage & $(1,18) 1.813$ & 0.195 \\
SCR of advantageous deck between blocks 1-2 & $(1,18) 1.096$ & 0.309 \\
SCR of advantageous deck between blocks 1-2 x P. stage & $(1,18) .172$ & 0.683 \\
\hline HR of risky deck between blocks 1-2-3 & $(1.752,36.782) 4.536$ & 0.021 \\
HR of risky deck between blocks 1-2-3 x P. stage & $(3.503,36.782) 0.956$ & 0.435 \\
HR of advantageous deck between blocks 1-2-3 & $(1.931,46.334) 6.505$ & 0.004 \\
HR of advantageous deck between blocks 1-2-3 x P. stage & $(3.861,46.334) 4.576$ & 0.004 \\
SCR of risky deck between blocks 1-2-3 & $(2,32) 4.926$ & 0.014 \\
SCR of risky deck between blocks 1-2-3 x P. stage & $(2,32) 2.077$ & 0.142 \\
SCR of advantageous deck between blocks 1-2-3 & $(1.865,33.577) 0.522$ & 0.586 \\
SCR of advantageous deck between blocks 1-2-3 x P. stage & $(1.865,33.577) 5.033$ & 0.014 \\
\hline
\end{tabular}




\section{HR in the ICGT1}

The mean HR in case of the risky deck in the ICGT1 increased significantly in the second block compared to the first $(F(1,23)=$ $10.553, p<0.05)$. Taking into consideration Piaget's stages, there were significant differences between the stages too $(F(2,23)=$ $13.986, p<0.05)$. The most obvious mean enhancement of the HR in case of the risky deck was by children in the FOS (from 91.54 to 95.86 beats per minute).

The HR for the advantageous deck across all stages increased significantly from the first to the second block of ICGT1 $(F(1,24)=10.250, p<0.05)$. When Piaget's stage is taken into account, the difference in mean HR of the advantageous deck between two blocks was significant too $(F(2,24)=4.151, p<0.05)$. The biggest mean enhancement of the HR in case of the advantageous deck was in the FOS group (from 91.70 to 97.30 beats per minute).

\section{SCR in the ICGT1}

We have not observed significant differences in mean SCR between two blocks of ICGT1 after a card selection from the risky $\operatorname{deck}(F(1,18)=1.575, p>0.05)$ and also not after a card selection from the advantageous $\operatorname{deck}(F(1,18)=1.096, p>0.05)$. However, the mean SCR in the second block in both groups of children is slightly higher after a card selection from the risky deck (2.12 microsiemens in COS, 1.71 microsiemens in FOS) compared to the advantageous deck (1.90 microsiemens in COS, 1.53 microsiemens in FOS).

\section{HR in the ICGT2}

The HR of risky deck significantly changed during the playing of the ICGT2 $(F(1.752,36.782)=4.536, p<0.05)$, but the changes between the groups were not significant $(F(3.503,36.782)=0.956, p>0.05)$. This indicates that all children in all stages considered risky deck as risky and as the game progressed they expected unfavorable consequences after a card selection from this deck.

Particularly notable are differences in mean HR after a card selection from the advantageous deck in the ICGT2 $(F(1.931,46.334)=$ $6.505, p<0.05)$. When Piaget's stage is taken into account, the differences in mean HR after a card selection from the advantageous deck are significant too $(F(3.861,46.334)=$ $4.576, p<0.05)$. We expected that this deck would be perceived as risky by younger children and as risky at first and then later as advantageous by older children. Therefore, we also expected differences between the groups in changes in the mean HR after a card selection from the advantageous deck. In children in the PS we observed that the mean HR of the advantageous deck in the third block ( 90.40 beats per minute) decreased in comparison to the second block (92.96 beats per minute), which may reflect the children's adverse expectations about the consequences after selecting a card from this advantageous deck. An additional reason for the differences between Piaget's stages in HR during the game is the way the children in the COS played ICGT2. Their strategy seems uncertain because in the third block the number of the card selections from the advantageous and the risky deck was about 
the same and their score was close to zero $(-1)$. In children in the COS we also found the greatest interindividual differences $(S D=$ 12.52).

\section{SCR in the ICGT2}

The mean SCR after a card selection from the risky deck changed significantly throughout playing the $\operatorname{ICGT2}(F(2,32)=$ $4.926, p<0.05)$. But comparing the COS and FOS, the changes in the mean SCR after a card selection from the risky deck were not significant $(F(2,32)=2.077, p>0.05)$. This means that children of both stages considered approximately equally the riskiness of the deck and were concerned about the consequences after a selection from this deck.

Comparing children from the COS and FOS, the SCR after a card selection from the advantageous deck in the ICGT2 changed significantly $(F(1.865,33.577)=5.033, p<$ 0.05 ). The possible uncertainty in playing the ICGT2 in children in the COS indicates that the children considered both decks to be about equally risky (score in the third block

Table 6 Comparison of the differences in HR and SCR between risky and advantageous deck during the game in each stage separately and comparison between stages

\begin{tabular}{|c|c|c|c|}
\hline & & $\mathrm{F}$ & Sig. \\
\hline \multirow{8}{*}{ ت } & PS - HR of advant. vs. risky deck x 1-2 blocks & $(1,6) 0.023$ & 0.884 \\
\hline & COS - HR of advant. vs. risky deck x 1-2 blocks & $(1,8) 4.644$ & 0.063 \\
\hline & FOS - HR of advant. vs. risky deck x 1-2 blocks & $(1,9) 0.927$ & 0.361 \\
\hline & HR of advant. vs. risky deck x 1-2 blocks x P. stage & $(2,23) 0.434$ & 0.653 \\
\hline & PS - SCR of advant. vs. risky deck x 1-2 blocks & - & - \\
\hline & COS - SCR of advant. vs. risky deck x 1-2 blocks & $(1,9) 10.857$ & 0.009 \\
\hline & FOS - SCR of advant. vs. risky deck x 1-2 blocks & $(1,9) 2.004$ & 0.190 \\
\hline & SCR of advant. vs. risky deck x 1-2 blocks x P. stage & $(1,18) 2.128$ & 0.162 \\
\hline \multirow{8}{*}{$\underset{ٍ}{\circlearrowright}$} & PS - HR of advant. vs. risky deck x 1-2-3 blocks & $(2,10) 0.298$ & 0.748 \\
\hline & COS - HR of advant. vs. risky deck x 1-2-3 blocks & $(2,18) 6.794$ & 0.006 \\
\hline & FOS - HR of advant. vs. risky deck x 1-2-3 blocks & $(2,14) 1.346$ & 0.292 \\
\hline & HR of advant. vs. risky deck x 1-2-3 blocks x P. stage & $(3.711,38.962) 2.311$ & 0.079 \\
\hline & PS - SCR of advant. vs. risky deck x 1-2-3 blocks & - & - \\
\hline & COS - SCR of advant. vs. risky deck x 1-2-3 blocks & $(2,18) 2.200$ & 0.140 \\
\hline & FOS - SCR of advant. vs. risky deck x 1-2-3 blocks & $(2,14) 3.552$ & 0.057 \\
\hline & SCR of advant. vs. risky deck x 1-2-3 blocks x P. stage & $(2,32) 2.634$ & 0.087 \\
\hline
\end{tabular}

Note. PS - Preoperational stage, COS - Concrete operational stage, FOS - Formal operational stage 
is -1 ), whereas at the end of the game children in the FOS already distinguished the advantageous and the risky deck correctly (score in the third block is 6.2). This results in differences in the successive changes in the SCR between children of stages.

In order to analyze the increasing difference in HR between the risky and advantageous deck during the game in each stage separately, we conducted $2 \times 2$ (resp. $2 \times 3$ ) repeated measures ANOVA (HR values for each of 2 decks x 2 blocks, resp. 3 blocks). We used the same analysis also with the SCR data. For observing whether differences among groups are significant, we included the between-subject factor of "Piaget's stage" into the analysis (Table 6).

In the ICGT1 we found differences in SCR between the advantageous and risky deck, which changed significantly over time (between first and second block) in the COS $(F(1,9)=10.857, p<0.05)$. In the ICGT2 in the COS we found differences in HR between the advantageous and risky deck, which changed significantly over time (between first, second and thirdblock $)(F(2,18)=6.794, p<0.05)$.

\section{Discussion}

In our research we were interested whether it was possible to observe somatic markers in children. We used the modified Iowa Gambling Task, where we expected that in the more complex part of the game younger children will opt for risky options, because they will find them more advantageous.

\section{Performance in the ICGT1 and ICGT2}

In the ICGT1 (the less complex part of the game) all children relatively quickly figured out the game and parameters of the decks.
Already in the first block they achieved a positive score and their preference for the advantageous deck persisted along the whole game. However, children in the PS achieved a relatively low score of about zero. This means that they did not prefer one of the decks significantly more than the other and they selected approximately the same number of cards from the advantageous and risky deck.

Children in the PS preferred the risky deck in the ICGT2. The way the children in the COS played the ICGT2 seemed to be wavering. Children in the FOS moderately preferred the risky deck at first, but in the third block they already achieved a positive score. After a few experiences with a loss, only children in the FOS distinguished correctly which deck is more risky. This means that predominantly children in the FOS were able to understand all three parameters of the decks and relations between them.

Statistical analysis shows that groups differed in scores achieved in individual blocks. Because in none of the groups there was significant difference within blocks in ICGT1 nor within blocks in ICGT2, the children likely created a strategy that has not changed much during the game.

\section{Somatic markers in the PS}

Logs from card selections from the ICGT1 show that some children chose cards alternately and did not prefer any of the decks. Also, the psychophysiological changes in the HR were approximately the same after a card selection from both decks in the ICGT1. One explanation is that children in the PS were too little motivated for playing the game. But if we disregard small motivation, we still cannot deny that the somato-sensoric pat- 
tern did not activate, thus, the somatic markers were absent (Bechara et al., 1996; Damasio, 1996; Crone et al., 2005).

The ICGT2 children in the PS did not select a card alternately and we also observed subtle differences in the anticipatory HR. In the second block, HR slowed down after a card selection from the risky deck more than after the selection from the advantageous deck. In the third block it was reversed. However, as the differences were not significant, it is possible that this is a result of a chance. Therefore, we cannot unerringly negate the argument of Bechara et al. (1996), Damasio (1996), Crone et al. (2005) that in children (in this case up to seven years of age) somatic markers are absent in the process of decision-making.

\section{Somatic markers in the COS}

Children in the COS in the ICGT1 already in the first block chose cards mainly from the advantageous deck. In the ICGT1 anticipatory HR was lower (which means stronger fear reaction) after card selection from the risky deck than after a selection from the advantageous deck but the difference was not significant.

We observed in the ICGT1 greater SCR increase after a card selection from the risky deck than the advantageous deck, and this difference is notable. Children in the COS achieved a greater score increase from first to second block when compared to FOS group. Observed somatic markers changes in children in the COS reflect their improving performance while playing ICGT1.

Differences in anticipatory HR and SCR in the ICGT2 also practically copied the behavior data. Although differences in SCR do not seem as considerable, the trend corresponds to our expectations that in children in the COS skin conductance increases more after a choice (according to their understanding of the situation) they hold to be risky. Approximately from the second block occur observable differences in somatic markers. Negative score in the second block means that children avoided choices from the advantageous deck (because they expected adverse consequences). In the third block of the ICGT2 the ratio of choices from the risky and advantageous deck was almost balanced and the difference in anticipatory SCR after a card selection decreased as well.

Mean age of children examined in the COS was 10.45 . Since differences in anticipatory HR during the game were significant and we observed different values for risky and advantageous decks and since differences corresponds to the deck preference, this phenomenon can be attributed to the activation of the somatic sensory pattern. In this case, we believe that from approximately 10 years on, respectively from the COS in healthy children, somatic markers are manifested more notably and perform their function. Our findings correspond to Crone et al. (2003) and Hooper et al. (2008), who say, that children up to 12 , respectively up to 9 years of age are able to solve the IGT.

When a child in the COS, based on his or her intuitive feelings, chooses a behavior that is objectively risky, it is not because of absence of somatic markers, but because this child cannot take into account all factors of the situation yet (and therefore may intuitively prefer also a risky option).

\section{Somatic markers in the FOS}

In the ICGT1 in children in the FOS the anticipatory HR was lower after a card selec- 
tion from the risky deck than after a selection from the advantageous deck. However, this difference was not significant.

At the beginning of the ICGT2 the difference in anticipatory HR between decks was relatively small (also the difference between the amount of cards selected from the risky and advantageous deck was minimal). In the second and third block the gap widened on behalf of the fear reaction after selecting from the risky deck. However, this difference was still small.

In the ICGT2 the risky deck is "misleading" because it contains only one losing card out of 10 cards. Given that children initially had very little experience with the loss, it is not surprising that they preferred a risky option at first. However, after several losses from the riskydeck, theyrealized all three parameters of the decks and somewhat began to prefer the advantageous deck in the third block. Since preference or avoidance of decks was relatively balanced, it is likely that the children were aware of all three characteristics of the decks, however, they considered both options as similarly risky at first. Therefore, the somatic markers did not manifest themselves after a card selection from one of the decks more than from another. Perhaps, if the children had the opportunity to play ICGT2 a longer time and had more experience with losses, they would have probably preferred the advantageous deck more often, which would have been reflected in the greater manifestations of somatic markers.

Received January 14, 2015

\section{References}

Bechara, A. (2007). Iowa Gambling Task, Professional Manual. Lutz, FL: Psychological Assessment Resources.
Bechara, A., Damasio, A., Damasio, H., \& Anderson, S. (1994). Insensitivity to future consequences following damage to human prefrontal cortex. Cognition, 50, 7-15. doi: 10.1016/00100277(94)90018-3

Bechara, A., Damasio, H., Tranel, D., \& Damasio, A. R. (2005). The Iowa Gambling Task and the somatic marker hypothesis: Some questions and answers. TRENDS in Cognitive Science, 9, 159162. doi:10.1016/j.tics.2005.02.002

Bechara, A., Damasio, H., Tranel, D., \& Damasio, A. R. (1997). Deciding advantageously before knowing the advantageous strategy. Science, 275, 1293-1295. doi: 10.1126/science. 275 . 5304.1293

Bechara, A., Tranel, D., Damasio, H., \& Damasio, A. R. (1996). Failure to respond autonomically to anticipated future outcomes following damage to prefrontal cortex. Cerebral Cortex, 6, 215 225. doi: 10.1093/cercor/6.2.215

Crone, E. A., Bunge, S. A., Latenstein, H., \& van der Molen, M. W. (2005). Characterization of children's decision making: Sensitivity to punishment frequency, not task complexity. Child Neuropsychology, 11, 245-263. doi: 10.1080/ 092970490911261

Crone, E. A., Somsen R. J. M., van Beek, B., \& van der Molen, M. W. (2004). Heart rate and skin conductance analysis of antecedents and consequences of decision making. Psychophysiology, 41, 531-540. doi: 10.1111/j.1469-8986.2004. 00197.x

Crone, E. A., Vendel, I., \& van der Molen, M. W. (2003). Decision-making in disinhibited adolescents and adults: insensitivity to future consequences or driven by immediate reward? Personality and Individual Differences, 34, 1-17. doi: 10.1016/s0191-8869(02)00386-0

Damasio, A. R., Everintt, B. J., \& Bishop, D. (1996). The somatic marker hypothesis and the possible functions of the prefrontal cortex [and Discussion]. The Royal Society, 351, 1413-1420. doi: 10.1098/rstb.1996.0125

Figner, B., \& Murphy, R. O. (2011). Using skin conductance in judgment and decision making research. In M. Schulte-Mecklenbeck, A. Kühberger, \& R. Ranyard (Eds.), A handbook of process tracing methods for decision research, a critical review and user's guide. New York: Psychology Press.

Hinson, J. M., Whitney, P., Holben, H., \& Wirick, A. K. (2006). Affective biasing of choices in gambling task decision making. Cognitive, Affective, 
\& Behavioral Neuroscience, 6, 190-200. doi: 10.3758/CABN.6.3.190

Hooper C. J., Luciana, M., Wahlstrom, D., Conklin, H. M., \& Yarger, R. S. (2008). Personality correlates of Iowa Gambling Task performance in healthy adolescents. Personality and Individual Differences, 44, 598-609. doi: 10.1016/j.paid. 2007.09.021

Iversen, S., Kupfermann, I., \& Kandel, E. R. (2000) Emotional states and feelings. In E. R. Kandel, J. H. Schwartz, \& T. M. Jessell (Eds.), Principles of Neural Science. New York: McGraw-Hill Companies.

Overman, W. H., Frassrand, K., Ansel, S., Trawalter, S., Bies, B., \& Redmond, A. (2004). Performance on the IOWA card task by adolescents and adults Neuropsychologia, 42, 1838-1851. doi: 10.1016/ j.neuropsychologia.2004.03.014

Piaget, J. (1976). The psychology of intelligence. New Jersey: Littlefield, Adams \& Co.

Pilárik, L., \& Sarmány-Schuller, I. (2009). Emotional intelligence and decision-making of female students of social work in the Iowa Gambling Task. Studia Psychologica, 51, 319-328.
Stemmler, G., \& Wacker, J. (2010). Personality, emotion, and individual differences in physiological responses. Biological Psychology, 84, 541-551. doi: 10.1016/j.biopsycho.2009.09. 012

Tchanturia, K., Liao, P., Uher, R., Lawrence, N., Treasure, J., \& Campbell, I. C. (2007). An investigation of decision making in anorexia nervosa using the Iowa Gambling Task and skin conductance measurements. Journal of the International Neuropsychological Society, 13, 635-641. doi: 10.10170S1355617707070798

Túnyiová, M., Sarmány-Schuller, I., Ibanez A., Bruno D., \& González Gadea, M. L. (2012). Rozhodovanie argentínskych a slovenských detí v Iowa Gambling Task. Sociálne procesy a osobnost' 2012: XV. ročník medzinárodnej vedeckej konferencie. Bratislava: Ústav experimentálnej psychológie SAV.

van den Bos, R., Homberg, J., \& de Visser, L. (2012). A critical review of sex differences in decisionmaking tasks: Focus on the Iowa Gambling Task. Behavioural Brain Research, 238, 95-108. doi: 10.1016/j.bbr.2012.10.002 